\title{
A New Deep Imaging Survey of Planetary Nebulae with known Binary Central Stars
}

\author{
S.A. Bell ${ }^{1}$ and D.L. Pollacco ${ }^{2}$ \\ ${ }^{1}$ Royal Greenwich Observatory, Cambridge, UK; \\ ${ }^{2}$ Isaac Newton Group, La Palma, Canary Islands
}

Binarity in central stars is often thought to be the most likely explanation for the bipolar and axisymmetric shapes seen in planetary nebulae. Theoretical computations show that low mass stellar companions - possibly even planets, merged nuclei and long period systems will also produce bipolar-shaped nebulae. As such all known short period binary central stars are expected to have an axisymmetric nebula surrounding them.

Since most of the pioneering work on this topic (Bond \& Livio 1990) was undertaken with a $0.9-\mathrm{m}$ telescope, we have re-imaged all known planetary nebulae with binary central stars using ESO's 3.5-m New Technology Telescope (NTT) using narrow-band filters. Most of these objects were discovered from samples of planetary nebulae that were known to have relatively faint nebula and bright central stars, a necessity bearing in mind that the original observations were made using photoelectric photometers. Despite this, Bond and Livio's results show that most objects were either elliptical or axisymmetric in shape and conformed to the notion that the planetary nebulae were simply ejected common envelopes.

The NTT images presented here confirm Bond and Livio's conclusions and show hitherto unseen nebular structures in many of the objects. For example, one object SuWt2, only recently found to contain a binary central star by Bond (1995), is shown to be a highly evolved bipolar nebula some 8-9 arcminutes in size. The original [N II] image of K1-2 shows that the "jets" are extremely tightly collimated with a length of some 20 arcseconds each. We have also obtained spectroscopy of this object which shows that the velocity of the material in the jet is low and hardly discernable from that of the nebula while the level of ionisation is significantly higher.

\section{REFERENCES}

Bond, H.E., Livio, M., 1990, Ap.J., 355, 568.

Bond, H.E., 1995, Ann. Israel Phys. Soc., 11, 61. 